\title{
AN ANALOGUE OF LUBESEDER'S THEOREM FOR FORMATIONS OF FINITE RINGS
}

\author{
Dedicated to the memory of Hanna Neumann
}

CARLTON CHRISTENSEN

(Received 15 January 1973)

Communicated by M. F. Newman

For formations of finite soluble groups, the properties of Frattini closure and local defineability are known to be equivalent (see [2]). The investigations of Barnes and Gastineau-Hills [1] on the other hand reveal that although every Frattini closed formation of finite-dimensional soluble Lie algebras over an algebraically closed field of zero characteristic is local, without the algebraic closure condition the relationship between the two properties breaks down even for supersoluble Lie algebras. We are concerned here with the analogous problem for rings. The main results are;

THEOREM 1. Let $\mathfrak{F}$ be a formation of finite rings and let $\pi$ be the set of primes dividing* $\mathfrak{F}$ then $\mathfrak{F}$ is a local formation if and only if it is Frattini closed and conains the formation of finite nilpotent $\pi$-rings.

THEOREM 2. There exist Frattini closed formations of finite rings that are not local.

It is clear from the example of Barnes and Gastineau-Hills mentiored above, that the analogous proposition to Theorem 1 is false for finite-dimensional soluble Lie algebras.

The terms "formation", "local" and "Frattini closed" used atove have natural definitions analogous to those used in group theory. Thus, following usual terminology, we refer to a class of rings as a homomorph whenever it contains all homomorphic images of its members and as a formation if in addition it is subdirect product closed. For any ring $R$, the intersection $\phi(R)$ of its maximal ideals, when such exist, is called the Frattini subring of $R$. It is well-known and

* A prime is said to divide a formation of finite rings if it divides the characteristic of at least one non-trivial ring in that formation. 
easy to prove that for finite rings $\phi(R)$ is contained in the Jacobson radical $J(R)$ of $R$. We are concerned here with classes of rings that contain a ring $R$ whenever they contain it Frattini factor ring $R / \phi(R)$. Such classes are said to be Frattini closed.

One of the most elementary non-trivial examples of a Frattini closed formation of rings is the class $\mathfrak{N}$ of finite nilpotent rings. This class can be described locally in the sense that $R \in \mathfrak{N}$ if and only if the minimal ideals of its factor rings $R / K$ are trivial left $R$-modules. More generally, we refer to the minimal ideals of the factor rings of a finite ring $R$ as chief factors of $R$. Since each chief factor has prime characteristic it can be classified, according to which prime $p$ is involved, as a $p$-chief factor. For any chief factor $H / K$ of $R$ we denote its left annihilator $\{r: r \in R \wedge r H \subseteq K\}$ in $R$ by $A_{R}(H / K)$.

Given a set of primes $\pi$ and a function $f$ with domain $\pi$ whose images are formations of finite rings, the class $\mathfrak{F}$ of $\pi$-rings whose $p$-chief factors $H / K$ have the property $R / A_{R}(H \mid K) \in f(p)$ for each $p \in \pi$ is a formation. This can be proved in a manner directly analogous to the corresponding result in group theory (see [2]). Such a formation is called the local formation defined by the formation function $f$ with support $\pi$.

In view of the primary decomposition of finite rings, it is easy to see that for

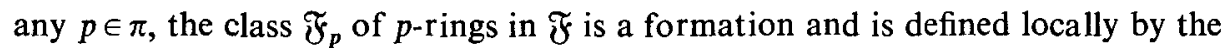
formation function $f_{p}$ with support $\{p\}$ and image $\{f(p)\}$. Moreover, $\mathfrak{F}$ is the direct product of the $\mathfrak{F}_{p}$ 's in the sense that $R \in \mathfrak{F}$ if and only if $R$ is a (ring) direct product of rings from the $\mathfrak{F}_{p}$ 's. The converse is equally true, that given a set of local formations $\left\{\mathfrak{F}_{p}: p \in \pi\right\}$ for some $\pi$ with $\mathfrak{F}_{p}$ defined by some formation function $f_{p}$, the direct product of the $\mathfrak{F}_{p}$ 's is the local formation defined by the formation function $f: p \leftrightarrow f_{p}(p)$ with support $\pi$. We refer to the $\mathscr{F}_{p}$ 's as the p-components of $\mathfrak{F}$. On the other hand, since the Frattini subring of a finite ring is the (ring) direct product of the Frattini subrings of its p-primary components, it is clear that a formation is Frattini closed if and only if its $p$-components are Frattini closed. Thus, as is usually the case with finite rings, it is sufficient for present purposes to consider only $p$-rings. We use the symbol $\mathfrak{P}$ to denote the local formation of finite $p$-rings.

The most elementary non-trivial local formations are the formations $\mathfrak{N}_{p}$ of finite nilpotent $p$-rings in the sense that they contain no proper local formations. That they are local is clear: indeed they are defined by the formation function with support $\{p\}$ whose solitary image is the formation of trivial rings. It follows that $\mathfrak{N}_{p}$ is contained in every local formation divided by $p$.

The following simple lemma is most useful. It is an immediate consequence of the characterisation of the Jacobson radical $J(R)$ as the annihilator of all chief factors of $R$. 
LEMMA. Let $\mathfrak{F}$ be the formation of finite p-rings defined locally by a formation function $f$ then $R \in \mathfrak{F}$ if and only if $R \in \mathfrak{P}$ and $R / J(R) \in f(p)$.

ProOF OF TheOREM 1. Let $\mathfrak{F}$ be a local formation of finite $p$-rings defined locally by a formation function $f$, then from the observations above, $\mathfrak{N}_{p} \subseteq \mathfrak{F}$. So let $R$ be a finite ring such that $R / \phi(R) \in \mathfrak{F}$. Then $R$ is a $p$-ring since $\phi(R)$ is a (ring) direct product of the Frattini subrings of the primary components of $R$, each of which is proper. Moreover, $J(R / \phi(R))=J(R) / \phi(R)$ so that, writing $\bar{R}$ for $R / \phi(R)$, the lemma yields $\bar{R} / J(\bar{R}) \in f(p)$. That is, $R / J(R) \in f(p)$ and hence $R \in \mathfrak{F}$ as required.

The sufficiency of the conditions of the theorem follows immediately from the next result.

Proposition 1. Let $\mathfrak{F}$ be a Frattini closed formation of finite p-rings containing $\mathfrak{N}_{p}$, then $\mathfrak{F}$ is defined locally by the formation function $f$ with support $\{p\}$ and image $\{R: R \in \mathfrak{P} \wedge R / J(R) \in \mathfrak{F}\}$.

That the class $f(p)$ defined in Proposition 1 is a formation is easily checked directly. We can say more however:

Proposition 2. Let $\mathfrak{H}$ be a homomorph of finite rings then the class of finite rings $\mathfrak{H}^{J}=\{R: R / J(R) \in \mathfrak{H}\}$ is a Frattini closed formation.

Proof. Let $R \in \mathfrak{S}^{J}$ and let $\theta$ be a homomorphism of $R$, then the simple components of $R \theta / J(R \theta)$ are also simple components of $R / J(R)$ and hence $R \theta / J(R \theta) \in \mathfrak{H}$. It follows that $\mathfrak{S}^{J}$ is a homomorph. Moreover, if $R / U$ and $R / V$ are in $\mathfrak{H}^{J}$ and $U \cap V=0$, then each simple component of $R / J(R)$ is a simple component of one of $(R / U) / J(R / U)$ or $(R / V) / J(R / V)$ and hence lies in $\mathfrak{H}$. It follows that $R / J(R) \in \mathfrak{S}$ and that $R \in \mathfrak{Y}^{J}$. Finally, let $R / \phi(R) \in \mathfrak{H}^{J}$. Then, since $\phi(R) \subseteq J(R), R / J(R) \in \mathfrak{H}$ and hence $R \in \mathfrak{H}^{J}$ as required.

Proof of Proposition 1. Firstly note that by Proposition 2, $\mathscr{F}^{J}$ is a formation and so clearly $f(p)=\mathfrak{F}^{J} \cap \mathfrak{P}$ is also a formation. Let $\mathfrak{F}^{*}$ be the formation defined locally by the given formation function $f$, then it follows immediately from the lemma that $\mathfrak{F} \subseteq \mathfrak{F}^{*}$. So let $R \in \mathfrak{F}^{*}$ and assume inductively that all rings of smaller order than $R$ in $\mathfrak{F}^{*}$ are in $\mathfrak{F}$. Then, since $\mathfrak{F}$ is Frattini closed, if $K$ is a minimal ideal of $R$ it cannot be contained in $\phi(R)$ because $R / K \in \mathfrak{F}$ by induction. It follows that $K$ is complemented directly in $R$ by a maximal ideal $L$. If $L \neq 0$, then $K \cong R / L$ and $L \cong R / K$ are in $\widetilde{F}$ and hence $R$ is in $\mathscr{F}$. On the other hand, if $L=0$ then $R$ is a simple $p$-ring. Either $R$ is nilpotent, in which case $R \in \mathfrak{N}_{p} \subseteq \mathfrak{F}$ or $R$ is not nilpotent in which case $J(R)=0$ and by the lemma $R \in \mathfrak{F}$.

Unlike the analogous situation in finite soluble group theory, the existence of suitable nilpotent objects in a Frattini closed formation is by no means assured. 
Indeed, we show now that some Frattini closed formations of finite rings contain no non-trivial nilfoent rings.

Given a homomorph $\mathfrak{H}$ of finite rings, we denote by $\mathfrak{H}^{\varphi}$ the class $\{R: R / \phi(R) \in \mathfrak{S}\}$ and by $\mathfrak{H}^{\delta}$ the subdirect product closure of $\mathfrak{H}$. In other words, $\mathfrak{S}^{\delta}$ is the intersection of all subdirect product closed classes of finite rings containing $\mathfrak{H}$. It is easy to see that $\mathfrak{H}^{\varphi}$ is the Frattini closure of $\mathfrak{D}$ and that $\mathfrak{H}^{\delta}$ consists of all finite subdirect products of rings in $\mathfrak{h}$.

Proposition 3. Let $\mathfrak{H}$ be a homomorph of finite rings, then $\mathfrak{H}^{\varphi}$ and $\mathfrak{H}^{\delta}$ are homomorphs. In particular, $\mathfrak{H}^{\delta}$ is a formation. Moreover, if any one of $\mathfrak{H}, \mathfrak{H}^{\varphi}$ and $\mathfrak{H}^{\delta}$ contains a non-trivial nilpotent ring, all three of them do.

PRoof. Let $R \in \mathfrak{H}^{\phi}$ and let $\theta$ be a homomorphism of $R$, then since $\phi(R) \theta \subseteq \phi(R \theta), R \theta / \phi(R \theta)$ is a homomorphic image of $R / \phi(R)$ and hence is in $\mathfrak{H}$. If, on the other hand, $R \in \mathfrak{S}^{\delta}$, then there exist ideals $K_{1}, K_{2}, \cdots, K_{n}$ of $R$ such that $\bigcap_{i=1}^{n} K_{i}=0$ and, for each $i, R / K_{i} \in \mathfrak{H}$. But $R \theta / K_{i} \theta$ is a homomorphic image of $R / K_{i}$ for each $i$ and so is also in $\mathfrak{H}$. Thus $R \theta \in \mathfrak{H}^{\delta}$.

Moreover, if $R$ is a non-trivial nilpotent ring and lies in $\mathfrak{H}^{\varphi}$ then $R / \phi(R)$ is a non-trivial nilpotent ring in $\mathfrak{H}$. If $R$ lies in $\mathfrak{S}^{\delta}$, then $R / K_{i}$ is a non-trivial nilpotent ring in $\mathfrak{H}$. Since $\mathfrak{H}$ is contained in both $\mathfrak{H}^{\varphi}$ and $\mathfrak{H}^{\delta}$ the proof is complete.

COROLlaRY. Let $\mathfrak{H}$ be a homomorph of finite rings containing no non-trivial nilpotent rings, then $\mathfrak{F}=\bigcup_{r \in Z^{+}} H^{(\varphi \delta) r}$ is a Frattini closed formation of finite rings containing no non-trivial nilpotent rings.

Proof of Theorem 2. In the corollary above, choose for $\mathfrak{H}$ the class consisting of all fields with two elements and all trivial rings. Then the formation $\mathfrak{F}$ is Frattini closed but is not local by Theorem 1 .

Proposition 2 gives a good idea of the size of the formation $\mathfrak{F}$ in the corollary above: it is clear that $\mathfrak{H}^{\varphi} \subseteq \mathfrak{F} \subseteq \mathfrak{Y}^{J}$.

\section{References}

[1] D. W. Barnes, and H. M. Gastineau-Hills, 'On the theory of soluble Lie algebras', Math. Zeitschr. 106 (1968), 343-354.

[2] B. Huppert, Endliche Gruppen I, (Springer-Verlag, 1967).

Department of Pure Mathematics

Australian National University

Canberra, A. C. T. 2600

Australia. 